Open Access

\title{
Correction to: Physicochemical properties of $M$. longissimus dorsi of Korean native pigs
}

\author{
Gye-Woong Kim and Hack-Youn Kim ${ }^{*}$
}

\section{Correction}

Upon publication of this article [1], it was noticed that during typesetting, the words 'Breeds' and 'Items' were accidentally placed next to each other in Tables $1,2,3$ and 4 whereas they should be discrete. Please see below the correct Tables.

Table 1 Proximate components of $M$. longissimus dorsi muscles in LYD and KNP breeds

\begin{tabular}{|c|c|c|c|c|}
\hline \multirow[t]{2}{*}{ Items } & \multicolumn{2}{|c|}{ Breeds } & \multirow[t]{2}{*}{ Overall mean } & \multirow[t]{2}{*}{$\mathrm{t}$-values } \\
\hline & LYD & KNP & & \\
\hline Moisture (\%) & $73.67 \pm 0.69$ & $74.06 \pm 0.18$ & $73.87 \pm 0.54$ & $2.12^{*}$ \\
\hline Crude fat (\%) & $2.03 \pm 0.67$ & $1.97 \pm 0.48$ & $2.00 \pm 0.57$ & $0.29^{\mathrm{NS}}$ \\
\hline Crude protein (\%) & $22.13 \pm 0.30$ & $21.45 \pm 0.60$ & $21.79 \pm 0.58$ & $3.91^{* * *}$ \\
\hline Crude ash (\%) & $0.72 \pm 0.01$ & $0.66 \pm 0.02$ & $0.69 \pm 0.03$ & $10.58^{* * *}$ \\
\hline
\end{tabular}

All values are the mean \pm standard deviation

${ }^{*} p<0.05,{ }^{* * *} p<0.001,{ }^{\text {NS }}$ Non-significant

Table 2 Physicochemical characteristics of M. longissimus dorsi muscles in LYD and KNP breeds

\begin{tabular}{|c|c|c|c|c|}
\hline \multirow[t]{2}{*}{ Items } & \multicolumn{2}{|c|}{ Breeds } & \multirow[t]{2}{*}{ Overall mean } & \multirow[t]{2}{*}{ t-values } \\
\hline & LYD & KNP & & \\
\hline Cooking loss (\%) & $34.46 \pm 1.68$ & $35.64 \pm 1.30$ & $35.05 \pm 1.60$ & $2.16^{*}$ \\
\hline $\mathrm{pH}$ & $5.56 \pm 0.10$ & $5.57 \pm 0.04$ & $5.57 \pm 0.07$ & $0.44^{\mathrm{NS}}$ \\
\hline CIE L* (lightness) & $53.52 \pm 2.47$ & $51.38 \pm 2.27$ & $52.45 \pm 2.57$ & $-2.47^{*}$ \\
\hline$a^{*}$ (redness) & $6.43 \pm 1.30$ & $10.40 \pm 2.27$ & $8.41 \pm 2.72$ & 5.88 \\
\hline b* (yellowness) & $3.27 \pm 0.97$ & $4.76 \pm 1.31$ & $4.01 \pm 1.36$ & $3.54^{* * *}$ \\
\hline
\end{tabular}

All values are the mean \pm standard deviation

${ }^{*} p<0.05,{ }^{* * *} p<0.001$, NS Non-significant
Table 3 Fatty acid composition of M. longissimus dorsi muscles in LYD and KNP breeds

\begin{tabular}{|c|c|c|c|c|}
\hline \multirow[t]{2}{*}{ Items } & \multicolumn{2}{|c|}{ Breeds } & \multirow[t]{2}{*}{ Overall mean } & \multirow[t]{2}{*}{ t-values } \\
\hline & LYD & KNP & & \\
\hline Myristic & $1.64 \pm 0.13$ & $1.35 \pm 0.34$ & $1.50 \pm 0.19$ & $6.40^{* * *}$ \\
\hline Palmitic & $25.30 \pm 0.76$ & $24.47 \pm 0.69$ & $24.88 \pm 0.83$ & $3.14^{* *}$ \\
\hline Palmitoleic & $3.10 \pm 0.20$ & $3.08 \pm 0.28$ & $3.09 \pm 0.24$ & $0.23^{\mathrm{NS}}$ \\
\hline Stearic & $14.46 \pm 1.57$ & $13.29 \pm 1.21$ & $13.88 \pm 1.50$ & $2.27^{* *}$ \\
\hline Oleic & $46.24 \pm 2.09$ & $43.87 \pm 2.59$ & $45.05 \pm 2.60$ & $2.75^{*}$ \\
\hline Vaccenic & $0.26 \pm 0.01$ & $0.14 \pm 0.14$ & $0.20 \pm 0.64$ & $30.16^{* * *}$ \\
\hline Linoleic & $7.23 \pm 0.66$ & $11.77 \pm 1.11$ & $9.50 \pm 2.48$ & $13.63^{* * *}$ \\
\hline g-Linoleic & $0.06 \pm 0.11$ & $0.05 \pm 0.06$ & $0.06 \pm 0.09$ & $2.46^{*}$ \\
\hline Linolenic & $0.40 \pm 0.32$ & $0.42 \pm 0.04$ & $0.41 \pm 0.04$ & $2.24^{*}$ \\
\hline Eicosenoic & $0.97 \pm 0.07$ & $1.13 \pm 0.06$ & $1.05 \pm 0.11$ & $6.99^{* * *}$ \\
\hline Arachidonic & $0.35 \pm 0.10$ & $0.41 \pm 0.04$ & $0.38 \pm 0.08$ & $2.38^{*}$ \\
\hline SFA $^{a}$ & $41.40 \pm 2.39$ & $39.11 \pm 1.88$ & $40.25 \pm 2.41$ & $2.91^{* *}$ \\
\hline USFA $^{\mathrm{b}}$ & $58.60 \pm 2.39$ & $60.89 \pm 1.88$ & $59.75 \pm 2.41$ & $2.91^{* *}$ \\
\hline MUFA $^{c}$ & $50.57 \pm 2.28$ & $48.22 \pm 2.75$ & $49.40 \pm 2.76$ & $2.54^{*}$ \\
\hline PUFA $^{d}$ & $8.04 \pm 0.72$ & $12.66 \pm 1.13$ & $10.35 \pm 2.53$ & $13.39^{* * *}$ \\
\hline MUFA/SFA & $1.23 \pm 0.12$ & $1.24 \pm 0.13$ & $1.23 \pm 0.12$ & $0.24^{\mathrm{NS}}$ \\
\hline PUFA/SFA & $0.20 \pm 0.02$ & $0.32 \pm 0.02$ & $0.26 \pm 0.07$ & $14.99^{* * *}$ \\
\hline
\end{tabular}

All values are the mean \pm standard deviation

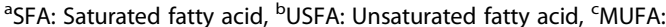

Monounsaturated fatty acid, dPUFA: Polyunsaturated fatty acid

${ }^{*} p<0.05,{ }^{* *} p<0.01,{ }^{* * *} p<0.001$, NS Non-significant

* Correspondence: kimhy@kongju.ac.kr

Department of Animal Resources Science, Kongju National University, Yesan, Chungnam 32439, South Korea 
Table 4 Sensory evaluation of $M$. longissimus dorsi muscles in LYD and KNP breeds

\begin{tabular}{|c|c|c|c|c|}
\hline \multirow[t]{2}{*}{ Items } & \multicolumn{2}{|c|}{ Breeds } & \multirow[t]{2}{*}{ Overall mean } & \multirow[t]{2}{*}{ t-values } \\
\hline & LYD & KNP & & \\
\hline Visual color & $8.25 \pm 0.40$ & $8.56 \pm 0.56$ & $8.41 \pm 0.48$ & $-1.55^{\mathrm{NS}}$ \\
\hline Flavor & $7.88 \pm 0.86$ & $8.79 \pm 0.62$ & $8.34 \pm 0.74$ & $-3.00^{* *}$ \\
\hline Tenderness & $9.08 \pm 0.29$ & $8.42 \pm 0.73$ & $8.75 \pm 0.51$ & $-2.92^{* *}$ \\
\hline Juiciness & $8.98 \pm 0.59$ & $7.90 \pm 0.64$ & $8.44 \pm 0.62$ & $-4.26^{* * *}$ \\
\hline fff-flavor & $8.67 \pm 0.50$ & $8.79 \pm 0.39$ & $8.73 \pm 0.45$ & $-0.52^{\mathrm{NS}}$ \\
\hline Overall acceptability & $8.13 \pm 0.68$ & $9.29 \pm 0.33$ & $8.71 \pm 0.51$ & $-5.34^{* * *}$ \\
\hline
\end{tabular}

Means and standard deviations were denoted by Likert's scale $(10=$ very excellent, $1=$ very poor)

${ }^{* *} p<0.01,{ }^{* * *} p<0.001,{ }^{\text {NS }}$ Non-significant $(p>0.05)$

The changes to these tables have been actualised by means of this Correction article.

Received: 4 April 2018 Accepted: 4 May 2018

Published online: 22 June 2018

\section{Reference}

1. Kim GW, Kim HY. Physicochemical properties of M. longissimus dorsi of Korean native pigs. J Anim Sci Technol. 2018;60:6. https://doi.org/10.1186/ s40781-018-0163-y. 